6 Iliffe S, Manthorpe J. The hazards of early recognition of dementia: a risk assessment. Aging Ment Health 2004; 8: 99-105.

7 Iliffe S, Wilcock J. Commissioning dementia care: implementing the National Dementia Strategy. J Integr Care 2009; 17: 3-11. the United States: is there evidence of the compression of cognitive morbidity? Alzheimers Dement 2008; 4: 134-44.

9 Langa KM, Llewellyn DJ, Lang IA, Weir DR, Wallace RB, Kabeto MU, et al. Cognitive health among older adults in the United States and in England. BMC Geriatr 2009; 9: 23.

10 Kramp VP, Herrling P. List of drugs in development for neurodegenerative diseases. Neurodegen Dis 2009; 6: 37-86.

\title{
Solution-focused psychiatry
}

\author{
Jan Martijn Bakker, ${ }^{1}$ Fredrike P. Bannink, ${ }^{2}$ Alasdair Macdonald ${ }^{3}$
}

The Psychiatrist (2010), 34, 297-300, doi: 10.1192/pb.bp.109.025957

${ }^{1} \mathrm{GGZ}$ InGeest, Haarlem, The Netherlands; ${ }^{2}$ Amsterdam, The Netherlands; ${ }^{3}$ Dorchester, UK

Correspondence to Alasdair Macdonald

(macdonald@solutionsdoc.co.uk)
Summary Solution-focused brief therapy (SFBT) can be widely implemented in psychiatric practice as a short form of psychotherapy that reinforces the client's autonomy and focuses on what the client wants instead of on the problem. It was developed by an iterative process of removal from existing therapy of any features not found to promote good outcomes for the attenders. Research indicates that SFBT is effective and cost-efficient, and when used in practice makes the psychiatrist's work more satisfying. It can be used as a primary intervention, for example during crisis intervention, as a formal psychotherapy and as an addition to pharmacotherapy.

Declaration of interest F.P.B. and A.M. both offer training and consultancy in solution-focused methods.
Brief treatments are in vogue. The focus is shifting to managed care and stepped care; clients are becoming increasingly emancipated and ask for efficient and respectful therapeutic interventions. Short forms of treatment include protocol-driven problem-focused (cognitive) behaviour therapy, with diagnosis and treatment aiming to reduce or stop the problem or complaint.

Solution-focused brief therapy (SFBT), in which efficiency and work satisfaction appear as important motivating factors, has been gaining popularity since the 1980s. In SFBT the focus is on determining and achieving the client's preferred future: what does the client want instead of their problem or complaint? In many areas of the world, (mental) healthcare services now work from a solution-focused premise.

This article introduces SFBT as an efficient addition to current psychiatric practice and holds its applicability up to the light, specifically from the psychiatrist's point of view. ${ }^{1}$

\section{What is SFBT?}

\section{Historical background}

Developed during the 1980s by deShazer, Berg and colleagues at the Brief Family Therapy Center in the USA, SFBT expands upon the findings of Watzlawick, Weakland and Fisch, ${ }^{2}$ who found that the attempted solution would often perpetuate the problem and that an understanding of the origins of the problem was not (always) necessary. deShazer emphasised the importance of building solutions rather than solving problems, and positioned the client in the role of an expert. ${ }^{3}$ The client is invited to reflect on what they would like to replace their problem with and at what stage they would consider the therapy a success.

\section{Goal formulation}

During the first conversation the client is asked to state their goal in positive, concrete and achievable behavioural terms: 'What needs to come out of this therapy? What do you want instead of your problem?' They may also be asked, 'What are your best hopes? What difference will achieving this goal make?'

Sometimes 'the miracle question' is put forward: 'Imagine a miracle occurring tonight that would (sufficiently) solve the problems which brought you here, but you will be unaware of this since you will be asleep. What would be the first sign tomorrow morning that would tell you that this miracle has taken place?' Next, the client is invited to describe how this day after the miracle would proceed, as elaborately and concretely as possible.

\section{Exceptions}

Solution-focused brief therapy starts from the assumption that one can always find exceptions to the problem: no 
problem or complaint is always present to the same extent. These positive exceptions, when the problem or complaint is less serious or not felt for a while, are often overlooked by the client or discarded as trivial due to their blinkered focus on the problem. The solution-focused therapist actually emphasises the exceptions and asks: 'At what times is the problem or complaint not there or is there to a lesser extent and what is different about those times? What do you do differently at those times?' The client may also be asked questions regarding the moments when the described miracle or preferred situation is already occurring to some extent and what they are then doing differently.

\section{Scaling questions and competence questions}

The client is invited to indicate to what degree their goal has already been achieved on a scale of $0-10$, with 10 being the most desirable outcome and 0 the worst things have ever been. "What did you do/What have you already done to reach this score? What will one point higher on the scale look like? What will you be doing differently then? What point on the scale do you want to reach for you to consider the goal (sufficiently) achieved? At what number would you see yourself as ready to conclude the therapy?'

\section{Client-therapist relationship: the visitor, the complainant and the customer}

In SFBT the therapist focuses on the client's motivation with respect to changing their behaviour. Three specific types of client-therapist relationships are distinguished: the visitor, complainant and customer-type relationship. The 'visitor' has been sent or referred by others and claims not to experience a problem, other than, possibly, some pressure from the person referring them. The 'complainant' is suffering emotionally but does not see themself as part of the problem and/or the solution: the other person or the world needs to change, rather than the client. The 'customer' does see themself as part of the problem and/ or the solution and is motivated to change their behaviour. By relating to the motivation of the client, the solutionfocused therapist is expert in applying those interventions that invite visitors and complainants to become customers.

\section{Feedback}

At the end of every conversation the solution-focused therapist formulates feedback for the client containing compliments and, depending on the therapeutic relationship, some homework suggestions. A customer is asked to carry out a behaviour assignment, for instance to do more of what brings their goal closer or to pretend that the miracle has already occurred. A complainant may be asked to undertake an observation assignment, for instance to pay attention to what is going well and is in no need of change. A visitor receives information but no suggestions, since they are not (yet) motivated to take action themselves.

\section{Therapist's attitude}

The attitude of the solution-focused therapist is one of not knowing: he allows himself to be informed by the client, whose own life context will determine in what way solutions are devised. Another aspect of the therapist's attitude is leading from one step behind. In this the therapist, metaphorically speaking, stands behind the client and taps them on the shoulder with solution-focused questions, inviting them to look at their preferred future and, in order to achieve this goal, to envisage a wide horizon of personal possibilities.

\section{Follow-up conversations}

In follow-up conversations the client and therapist carefully explore what has improved. 'What has been better since we last met?' is an invaluable opening to any contact, even if the client has been attending for many years. The therapist asks for a detailed explanation of the positive exceptions, gives compliments and emphasises the client's personal input in finding solutions. At the end of every conversation the client is asked whether they feel another meeting is still necessary, and if so, when they would like to return. In fact, in many cases the client feels it is not necessary to return or schedules an appointment further into the future than is typical in therapy.

\section{Evidence}

Stams et $a l^{4}$ carried out a meta-analysis of 21 international outcome studies. The results demonstrate a modest and positive effect of SFBT, at the same level as other forms of therapy. Interventions in outcome studies that were carried out more recently turn out to be the most effective; according to the authors this is likely due to a better execution of the technique. They conclude that SFBT is as effective as traditional forms of therapy. However, SFBT achieves a positive effect in less time and encourages the autonomy of the client. Similar findings emerged from Kim's meta-analysis of 22 studies. $^{5}$ In the overview of outcome studies by Macdonald ${ }^{6}$ (update available at www.solutionsdoc.co.uk), 80 evaluation studies have extended from 2 weeks to 6 years, and include 2 metaanalyses, 9 randomised controlled trials and 27 comparison studies. Comparison treatments have included short-term and long-term psychodynamic therapy, cognitive-behavioural therapy and programmes for substance misuse. The findings show that, like other psychological therapies, SFBT is effective for more than $60 \%$ of cases and that, unlike other therapies, SFBT has been shown to be equally effective for all social classes. The therapy is used within intellectual disability services, education and the criminal justice system, including domestic violence.

\section{Psychiatrist and SFBT}

\section{Indications and contraindications}

Solution-focused brief therapy is suitable for virtually all work environments as a 'monotherapy' or in combination with a problem-focused therapy. Depending on the nature of the complaint an essentially problem-focused approach may be chosen (e.g. pharmacotherapy), in which the supplementary use of SFBT is often valuable. It is wrong to assume 
that SFBT can only be applied to 'lighter' problems O'Hanlon \& Rowan describe how SFBT is applied to chronic and severe mental illnesses such as psychotic disorders. ${ }^{7}$

Because SFBT does not require a formal structure, it can be useful even in a busy out-patient clinic (all three authors work in such settings). The attitude of the therapist, attention to goal formulation by the client and 'tapping' into the often surprisingly large arsenal of competencies possessed by the client and their environment appear to be key elements in a successful outcome. Both the attender and the treatment team may contribute goals to the process and some incompatibilities may need to be acknowledged or negotiated. The therapy is also suitable for treating addiction-related problems, partly due to the considerable attention paid to the client's motivation to change their behaviour. $^{8}$

Can SFBT also be applied to Axis II disorders? The answer is yes, or rather, the question is incorrectly posed, as it implies that the goal is to make the respective mental disturbance disappear. However, SFBT asks the client what their goal is, which in practice often turns out to be a different, more achievable goal than the one the therapist has in mind.

Contraindications for SFBT are: the situation where it is impossible to establish a dialogue with the client, a wellexecuted solution-focused therapy that has yielded disappointing results, or the situation where the therapist is not prepared or unable to let go of their attitude as an expert.

\section{Diagnosis}

Solution-focused brief therapy is a form of treatment that requires no extensive diagnosis. One may choose to commence treatment immediately and, if necessary, pay attention to diagnosis at a later stage. Severe psychiatric disorders or a suspicion thereof justify the decision to conduct a thorough diagnosis, since the tracing of the 'underlying' organic pathology, for instance, has direct therapeutic consequences.

Out-patients in primary or second-line healthcare are suitable for a solution-focused approach. During the first or follow-up conversation it will automatically become clear whether an advanced diagnosis will be necessary, for example, if there is a visible deterioration in the client's condition or if the treatment fails to give positive results. Analogous to stepped care, one could think of stepped diagnosis.

\section{Practice guidelines and protocols}

Diagnosis-oriented practice guidelines do not yet mention SFBT. However, if a customer-type relationship is absent, working according to guidelines or protocols will be difficult, since the client is not (yet) motivated to undertake congruent assignments. Solution-focused brief therapy can contribute to changing the therapeutic alliance from a complainant-type relationship to a customer-type relationship, which may be followed by protocol-driven, problemfocused interventions or further solution-focused therapy. Solution-focused brief therapy can be regarded as a form of behaviour therapy that takes as starting points the preferred behaviour and functional cognitions, rather than the problem behaviour and dysfunctional cognitions. ${ }^{9}$ Since 2006, the Dutch Association for Behavioural and Cognitive Therapy has included a Solution-Focused CognitiveBehavioural Therapy Section.

\section{Medication aspects}

Biological treatments applied by psychiatrists seem to be strictly problem-focused. Nevertheless, it does make a difference if the client has the idea that 'the depression will disappear' or that they will become 'energetic, active or relaxed'. A solution-focused approach to pharmacological treatment may consist of encouraging the client to give a detailed description of what the first signs of recovery might look like, assuming that the medication takes effect, and of how the recovery will further manifest itself. The clients are asked what they themselves can add to the effect of the medication, or what they can do to create a conducive environment in which the medication can have the maximum effect in helping them to pull through.

\section{Crisis intervention}

Solution-focused brief therapy often proves very useful in crisis intervention. The available time does not usually lend itself to an elaborate diagnosis and, further to this, a client in crisis benefits from regaining confidence in their personal competences and a future-oriented approach. Think for example of questions such as: 'How do you manage to carry on? What has helped you in the past weeks, even if only slightly?' Commonly, the client relinquishes competencies to the therapist ('you tell me what I should do'), a pitfall that can be avoided with SFBT. ${ }^{10}$

\section{Work satisfaction}

With his inquisitive attitude of not knowing, the therapist encourages the client to take action. To the greatest extent possible conversations focus on the client's envisaged future, on the stage the client is already at and on what further steps they might take to make further progress. In SFBT the client tends to do most of the work, which benefits both therapist and client. Frustration on the part of the therapist ('client shows resistance') and of the client ('the therapist does not understand me') is avoided when the therapist relates to the existing motivation of the client and makes sure not to approach a visitor or complainant as a customer during the conversations or to give them behavioural assignments as homework. ${ }^{11,12}$ A basic training in SFBT for a healthcare professional usually requires 2040 hours of teaching followed by supervision for several months thereafter.

Clients and therapists usually experience SFBT as a pleasant form of therapy. The invitation to describe the preferred situation in the future and the client's experience of their own competencies make the conversations lighter and more positive than problem-focused conversations. In this way SFBT also reduces the possibility of 'burn-out' for all those using the approach, including psychiatrists. 


\section{Conclusions}

Solution-focused brief therapy goes beyond the necessity for an extensive diagnosis, meets societal demands for efficiency, reinforces the competence and autonomy of the client and makes the work of the therapist more satisfying. By supplementing the 'classic' problem-focused approach with SFBT, this form of treatment becomes widely applicable in the psychiatric practice.

\section{About the authors}

Jan Martijn Bakker is a Psychiatrist working for GGZ InGeest, Haarlem, The Netherlands, Fredrike Bannink is a Clinical Psychologist in private practice, Amsterdam, The Netherlands, and Alasdair Macdonald is a Consultant Psychiatrist, Dorchester, UK.

\section{References}

1 Bakker JM, Bannink FP. Oplossingsgerichte therapie in de psychiatrische praktijk. [Solution focused therapy in psychiatric practice.] Tijdschr Psychiatr 2008; 50: 55-9.

2 Watzlawick P, Weakland JH, Fisch R. Change. Principles of Problem Formation and Problem Resolution. WW Norton, 1974.
3 deShazer S. Keys to Solution in Brief Therapy. WW Norton, 1985.

4 Stams GJJ, Dekovic M, Buist $K$, de Vries L. Effectiviteit van oplossingsgerichte korte therapie. Een meta-analyse. [Efficacy of solution-focused brief therapy. A meta-analysis.] Gedragstherapie (Dutch Journal of Behavior Therapy) 2006; 39: 81-94.

5 Kim JS. Examining the effectiveness of solution-focused brief therapy: a meta-analysis. Res Social Work Pract 2008; 18: 107-16.

6 Macdonald AJ. Solution-Focused Therapy: Theory, Research and Practice. Sage, 2007.

7 O'Hanlon B, Rowan T. Solution-Oriented Therapy for Chronic and Severe Mental IIIness. WW Norton, 2003.

8 Berg IK, Miller SD. Working with the Problem Drinker. A Solution-Focused Approach. WW Norton, 2007.

9 Bannink FP. Oplossingsgerichte vragen. Handboek oplossingsgerichte gespreksvoering. [Solution Focused Questions. Handbook Solution Focused Interviewing.] Pearson, 2006

10 Bannink FP. Posttraumatic success. Solution focused brief therapy. Brief Treat Crisis Interv 2008; 7: 1-11.

11 Bannink FP. Gelukkig zijn en geluk hebben. Zelf oplossingsgericht werken. [Being Happy and Being Lucky. Solution Focused Self-Help.] Pearson, 2007.

12 Bannink FP. Solution-focused brief therapy. J Contemp Psychother 2007 37: 87-94. 